\title{
Essential and current methods for a practical approach to comparative neuropathology
}

\author{
D. De Biase, O. Paciello \\ Department of Veterinary Medicine and Animal Production, Unit of Pathology, University of Naples Federico II, Napoli, Italy
}

[Received 24 September 2014; Accepted 2 October 2014]

\begin{abstract}
The understanding of mechanisms that provoke neurological diseases in humans and in animals has progressed rapidly in recent years, mainly due to the advent of new research instruments and our increasing liability to assemble large, complex data sets acquired across several approaches into an integrated representation of neural function at the molecular, cellular, and systemic levels. Nevertheless, morphology always represents the essential approaches that are crucial for any kind of interpretation of the lesions or to explain new molecular pathways in the diseases. This mini-review has been designed to illustrate the newest and also well-established principal methods for the nervous tissue collection and processing as well as to describe the histochemical and immunohistochemical staining tools that are currently most suitable for a neuropathological assessment of the central nervous system. We also present the results of our neuropathological studies covering material from 170 cases belonging to 10 different species of mammals. Specific topics briefly addressed in this paper provide a technical and practical guide not only for researchers that daily focus their effort on neuropathology studies, but also to pathologists who occasionally have to approach to nervous tissue evaluation to answer questions about neuropathology issues. (Folia Morphol 2015; 74, 2: 137-149)
\end{abstract}

Key words: histology, brain, histochemistry, immunohistochemistry, necropsy, nervous tissue fixation procedures, nervous tissue artefacts

\section{INTRODUCTION}

The delicate nature of nervous tissue tends to cause a large number of artefacts that significantly interfere determination of the correct diagnosis. Those artefacts in the nervous tissue may appear during the necropsy evaluation, the handling and the sampling of the material for the histological assessment. Thus, working with the nervous system requires accurate technical methods that pathologist sand researchers must know and possibly follow in order to provide high-quality material for macrosco- pic and histological evaluation. Moreover, histochemical special stains as well as immunohistochemistry and immunofluorescence are a fundamental component of neuropathology. Special stains are indeed necessary to show the pathological modifications that affect the neurons and the unique populations of cells composing the central nervous system (CNS). Here, we summarise useful information for a practical approach to the nervous system reporting our experiences and results studying some interesting cases of CNS pathology.

Address for correspondence: O. Paciello, DVM, PhD, Ms Infectious Diseases, Department of Veterinary Medicine and Animal Production, University of Naples Federico II, Via Delpino, 180137 Naples, Italy, tel: 00390812536466 office, tel: 00390812536470 lab, fax: 00390812536186, e-mail: paciello@unina.it 


\section{NEUROPATHOLOGICAL TECHNIQUES}

\section{Necropsy techniques}

The CNS is protected by a solid bony calvaria and by the vertebra. Thus, the skull and vertebral column have to be opened by considerable mechanical force to access the delicate CNS structures [23]. The brain and the spinal cord have to be carefully handled in order to avoid the many possible resultant artefactual changes. Excessive pressure or stretching should not be placed on the brain or the spinal cord. Additionally, the latter has not to be bent or folded during excision from vertebral canal.

Removal of the brain. The soft tissues of the neck have to be removed. Then, the animal can be decapitate after extending the neck by cutting ventrodorsally through the exposed atlanto-occipital joints. At this point, the head can be completely removed, separating the brain from the spinal cord. All the skin, muscles and soft tissues have to be removed from the head in order to expose the calvaria. The head is blocked by a vise to simplify the subsequent operations. To open the cranial vault dorsally, it has to be preferably used an electric Stryker saw and cutting across a transverse line behind the orbits and two lateral lines just medial to the occipital condyles while avoiding contact with the underlying brain tissue (Fig. 1A, C). Then the head is turn upside-down, tilted to one side and shake gently to detach the brain from the skull and to expose the remaining connection that has to be cut (Fig. 1B, D); finally, the brain can be completely extracted by gently shaking. This technique may be essentially applied for the removal of the brain of every mammalian species. However, it is clear that there are some differences between each species, mainly due to the dimensions and weight of the animals. For laboratory animals (e.g. small rodents), the tiny dimensions make the opening of the cranial cavity much easier. A crosscut at the level of nasal septum can be made using scissors and from this point, subsequently, the occipital and parietal bone can be cut, paying attention that the scissors do not penetrate the nasal cavity too deeply (Fig. 1E, F). Afterwards, the skull is removed and the brain and meninges can be seen.

The cranial vault, meninges, pituitary gland and fossa, the cranial nerves and their foramina have to be always examined immediately after removal of the brain to detect any relevant abnormalities [23]. The brain itself should be evaluated immediately after the removal to identify relevant macroscopic lesions. After fixation, another detailed examination follows transversal sectioning of the brain.

Removal of spinal cord. The removal of the entire spinal cord could be really onerous, especially in large animals. Alternatively, the pathologist could choose to isolate just the segment of the spinal cord that is involved by a pathologic change, especially if adequate clinical information inclusive of $X$-ray, magnetic resonance imaging (MRI), computed tomography imaging are disposable. After exposing the paraspinal muscles, the dorsal aspect of the vertebrae at the lumbosacral junction have to be removed. Then, proceeding cranially, the laminae of each consecutive vertebra has to be cut using an electrical Stryker saw paying attention to not touch the cord. Finally, the dorsal part of the vertebra can be removed cutting the nerve roots on each side. Once the spinal cord is exposed it can be progressively lifted out of the vertebral canal (Fig. 1G, $\mathrm{H}$ ).

For small laboratory animals, Meickle and Martin [12] suggested an easy method to collect the spinal cord by using a $6 \mathrm{~mL}$ or $12 \mathrm{~mL}$ syringe filled with tap water and attached to a short blunt needle of diameter (18 to 22 gauge). After removing the head, the spine has to be sever at the level of the intervertebral disc in the middle to caudal lumbar region. Then, the needle has to be insert in the vertebral canal giving 2 to 3 gentle pushes on the plunger followed by a very firm and rapid push. The spinal cord should pop out onto the working surface intact and with very few histologic artefacts. This method is optimal just for animals dead for no more than a few hours because the autolysis causes too much tissue softening for this procedure. This approach has also been successfully adapted to much larger animals [6].

\section{Fixation procedures}

Before processing and trimming, the nervous tissue has to be immersed in the fixative for an adequate length of time. Generally, a complete fixation takes between 5 and 10 days for small and large animal brains, respectively. A good practice is to leave the tissues in the original fixative solution for at least $24 \mathrm{~h}$ and then replace the original with fresh fixative and retain for at least another $24 \mathrm{~h}$ prior to trimming and processing. For routine diagnostic neuropathology evaluation, the immersion of the brain and/or the spinal cord in 10:1 buffered formalin solution to tissue is optimal. A single sheet of adsorbent paper between 

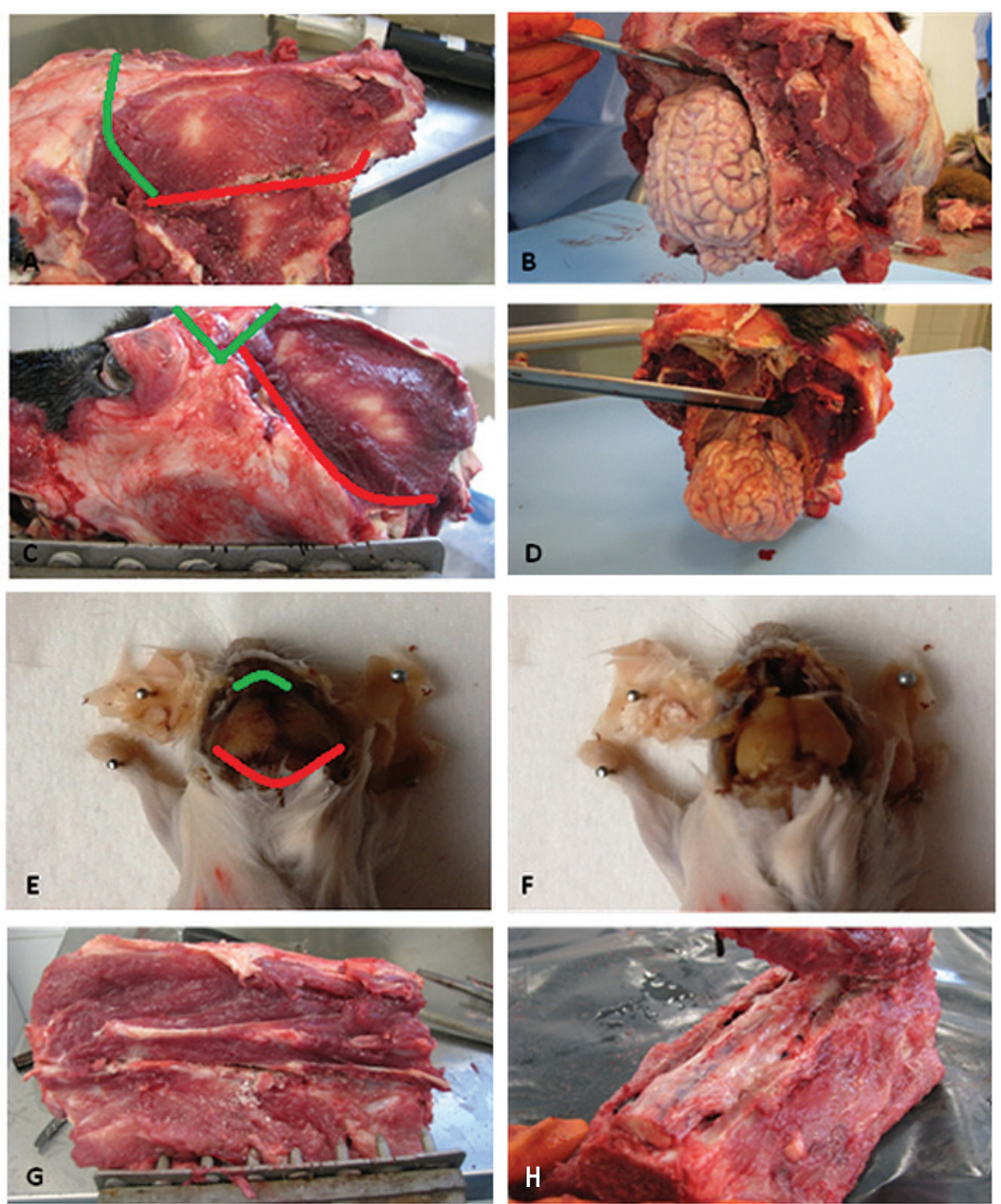

Figure 1. Necropsy technique in different species. Opening of the skull of a large animal (A, B) and of a dog (C, D). The marked lines indicate the direction of the cuts. $\mathbf{E}$ and $\mathbf{F}$ show the opening of the skull of a small rodent; $\mathbf{G}$. Removal of the lumbar segments of the spinal cord of a large animal. An electric saw is recommended to cut bilaterally the vertebral laminae; H. Removal of the dorsal part of the vertebral canal exposing the spinal cord. the brain and the bottom of the container can prevent adherence of the brain and severe artefactual changes. Although 10\% neutral buffered formalin is adequate for routine fixation of nervous tissue, improvements in quality can be achieved by using solutions of higher purity and better cross-linking ability. For this purpose, paraformaldehyde and/or glutaraldehyde are frequently used. Paraformaldehyde should be prepared fresh on the day of use if possible. If this is not possible, then it should be prepared the day before, refrigerated overnight, and brought to room temperature prior to use [4].

For laboratory animals, whole body perfusion through the vascular system is the preferred way to fix the nervous system because it provides the accurate morphological detail required for experimental studies and it's also necessary for investigations involving electron microscopy [4]. The concept for whole body perfusion is simply to replace the entire blood volume with a fixative solution through the vascular system while it remains patent [4]. The use of immunohistochemistry may dictate modification of fixation and/or perfusion procedures. In many cases, fixative procedures may be optimised for each individual staining procedure [20]. Tissue fixation methods alter the immunohistochemical demonstrability of neurofilament proteins, synpatophysin, and glial fibrillary acidic protein in human cerebellum [20]. For example, zinc aldehydes can be used as perfusion solutions for light microscopic evaluation of immunohistochemical reactions [15] and the demonstration of neuroglial cells may be best accomplished after perfusion with a $1 \%$ sodium sulfide solution followed by $4.0 \%$ paraformaldehyde [14].

\section{Macroscopic evaluation and sampling}

After fixation, transverse sections of the brain have to be obtained in order to examine all the main regions for gross evaluation. Pathological alterations may involve all structures in size (e.g. aplasia, hypopla- 


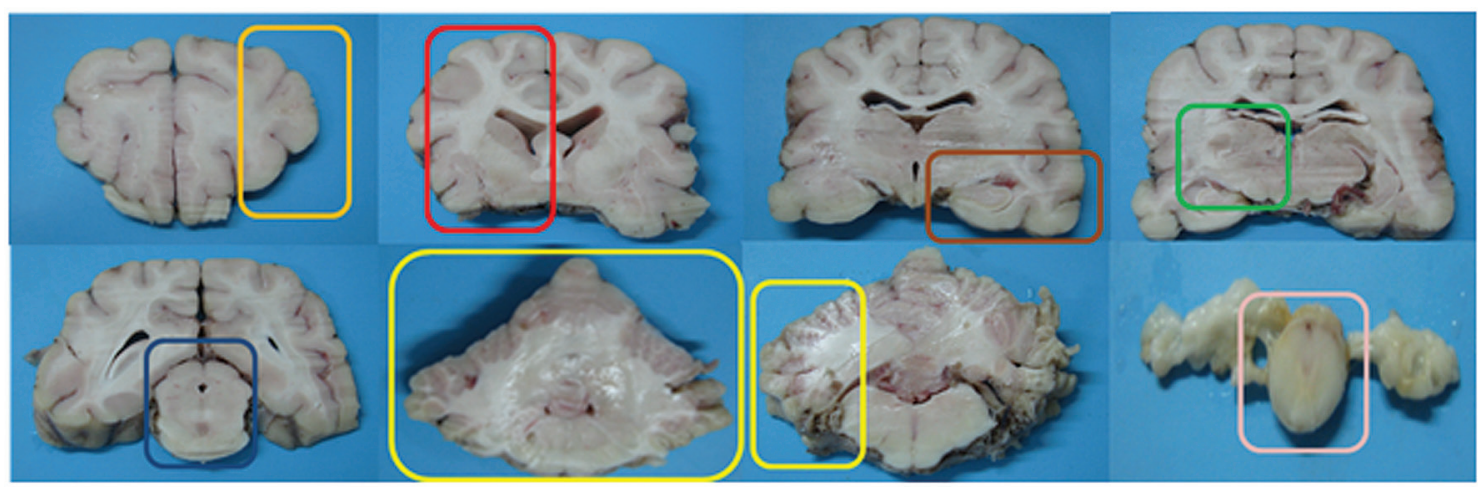

Figure 2. Representative sampling. Serial sections of a fixed canine brain. The orange box includes the area of frontal cortex, the red box includes the area of basal nuclei, the brown box includes the hippocampus, the green box includes the area of thalamus, the blue box includes the midbrain, the yellow boxes include the cerebellar cortex and the pink box includes the spinal cord.

sia, atrophy, swelling), shape (e.g. cerebellum coning) and symmetry of both sides of the brain. Stenosis, dilatation, compression and exudates involving the ventricular system may be found as well as swelling and congestion of the choroid plexus. Particularly relevant are the space-occupying masses that can have an inflammatory (e.g. abscess) or neoplastic origin.

Since mild or even severe damages of the nervous system may be not associated to macroscopic findings, the sampling for histological examination should be representative for all the main regions of the CNS (specifically: frontal cortex, area of basal nuclei, hippocampus, thalamus, midbrain, cerebellar cortex and spinal cord) (Fig. 2). However, it is clear that when a specific localisation is suggested by clinical examination or confirmed by MRI the sampling should involve even the damaged area.

Laboratory animals are generally used for experimental studies in which the target cell or brain area are well known. Plus, the tiny dimensions of the brain of these animals allow to survey the main region of the CNS all at once. Usually, a parasagittal section of brain (longitudinal section lateral to the centre line) from each animal, combined with a hemi-coronal section from the contralateral one-half brain is optimal for a rapid screening. Otherwise, when the specific CNS targets are unknown, it's recommended the evaluation of 4 coronal sections including cerebral cortex, hippocampus and cerebellum [6].

\section{Artefactual alterations}

The CNS is susceptible to some artefacts resulting from an improper handling, fixation or collecting of the samples that may simulate pathological conditions. Thus, the understanding at least of the most common artefacts is utmost important for a correct interpretation of the histological specimen. Two of the most frequently encountered artefacts are basophilic (dark) neurons and mucocytes (also known as Grynfeltt or Buscaino bodies) [4].

Dark neurons are usually the result of an excessive pressure on the tissues during the removal or the handling of the brain before fixation. Dark neurons have angular to irregular profiles and corkscrew-shaped dendrites. Both the nucleus and the cytoplasm shrink and stain deeply [4]. Dark neurons may be interpret as an early stage of degeneration, but it's relatively simple to distinguish them from neurons in advanced stage of necrosis that typically have a strongly eosinophilic cytoplasm.

Mucocytes (Buscaino bodies) are a rare artefact seen typically within the white matter of the brain and spinal cord. They are pale, blue-grey, periodic acid-Schiff (PAS) positive and metachromatic amorphous bodies and seem to arise by an unusual reaction between fixative solution and myelin. Dissolution of these bodies may occur, leaving the white matter spongiotic [21].

Myelin is particularly prone to artefact because it has a very high lipid content and reduced opportunity for protein cross-linking by chemical fixatives [4]. The solvent for paraffin embedding, especially ethanol, may produce fine vacuoles in white matter resembling a spongiotic state. This alteration is particularly evident if the tissues undergo autolytic change before fixation [21].

Brain and nerves are also particularly susceptible to the formation of pressure imprints when placed in close contact with small sponges used to hold tissues in embedment cassettes [7]. 


\section{Staining tissues of the CNS}

Haematoxylin and eosin (H\&E) stain display the cellular features providing a first evaluation of CNS tissues. However, H\&E-stained sections are not suitable to have useful information about the cytoplasm of neurons or the glial cells, even though expert neuropathologists can recognise different cell-types by the shape of their stained nuclei. The cells of the CNS are very unique and the complexity of their normal and pathological features makes the histochemical special stains a fundamental component of neuropathology. Special stains are indeed required to show the subtle details of neuronal cell body, axonal processes and myelin sheath and, furthermore, they are essential to define neuroglial cells.

The general condition of neurons can be evaluated by performing the cresyl violet stain that identify the neurons within the nervous tissue and demonstrate the loss of Nissl substance (chromatolysis) as a response to axonal injury [2]. Myelin sheath may be demonstrated by luxol fast blue (LFB) stain that is commonly used to detect demyelination in the CNS [2]. LFB stain is often used in combination with other stains such as cresyl violet, PAS and Holmer silver nitrate [2]. Combined LFB/cresyl violet can be useful for the demonstration of both myelin sheath and Nissl substance in tissue sections. LFB/PAS allows a correlative study of the cellular elements, fibre pathways, and vascular components of the nervous system [2]. Furthermore, it allows to show the basement membranes, the senile plaques, fungi and the presence of "corpora amylacea", small hyaline masses of unknown significance, but frequently observed with advancing age. LFB — Holmes silver nitrate stain allows to highlight both myelin sheath and nerve fibres in the same tissue section.

Glial fibres may be stained with Mallory's phosphotungstic acid haematoxylin (Mallory's PTAH) [9]. Unfortunately, Mallory's PTAH dyes very weakly the glial fibres and the fact that it also stains the myelin make it not specific and very difficult to interpret. The Holzer method is preferred over the Mallory's PTAH because it's more specific and allows the demonstration of areas of gliosis in addition to the glial fibres. However, both stains are not routinely used anymore for their lack of specificity and they have been largely replaced by immunochistochemical methods.

Silver impregnation techniques [22] make use of the argyrophilic properties of the cells to detect both the neuronal cell body and the axonal processes. Argyrophilia (from greek 'argyros' meaning 'silver' and 'philia' meaning 'attachment') is the ability of cells to bind silver salts; the precipitation of the salts occurs in the presence of a reducing agent [22]. Silver-staining methods are widely used for histopathological diagnosis, mainly for histological identification of pathological deposits [21] such as senile plaques and neurofibrillary tangles that are distinctive features of Alzheimer's disease and other tangle-bearing disorders. Currently, 4 major silver-staining methods are routinely performed for neuropathological assessment: modified Bielschowsky, Bodian, Gallyas (GAL) and Campbell-Switzer method [22]. GAL method is undoubtedly one of the most recent advances in silver-staining methods. However, it's very complicated to establish which one of these stains is more useful, especially in neuropathology. Comparative studies of different silver-staining methods pointed out that the argyrophilic profiles are dependent not only on the staining method, but also on the target lesion. For this reason, it's preferred to combine a silver-stain to an immunohistochemical analysis in order to provide a more detailed and multifaceted view, which may allow more precise morphological delineation of disease-specific deposits [22].

Golgi impregnation is a histochemical stain, based on metallic impregnation of neurons and glial cells which has been in use for more than a century for studying neuronal and glial morphology and neuroanatomical connections in the brain [19]. Several modifications to the original stain have been tried. Although some of these modifications somewhat improved the quality of staining and others reduced time taken for staining, consistency and reproducibility of results were still unsatisfactory. In addition to lack of uniform staining, consistency and reproducibility, this method is also time-consuming, which practically limits its use as the first choice, particularly for the study of formalin-fixed brain tissue [24]. It's evident, nowadays, that immunohistochemistry techniques using specific markers are the most reliable tool to label neuroglial cells and processes.

Microglia in normal nervous tissues may be specifically demonstrated using lectin, a carbohydrate - binding proteins, combined to a highly specific avidin-biotin peroxidase marker system [10].

In neuropathology, selective histochemical stains are an essential tool to study chemical components, 
pathological inclusions or microorganism within cells and tissues. PAS is mainly used for staining structures containing carbohydrate macromolecules (glycogen, glycoprotein, proteoglycans). PAS staining can provide supplemental information for the diagnosis of several pathological conditions such as Glycogen storage diseases, to detect inclusions in ceroid lipofuscinosis or fungal infection, but only on living fungi; in contrast, Grocott's methenamine silver stain (GMS) will stain both living and dead fungal organisms [17].

Gram staining is a method of differentiating bacterial species in two large groups (Gram positive or Gram negative) depending on the properties of bacterial cell wall.

Amyloid deposition is often associated with several diseases such as Alzheimer, Parkinson, transmissible spongiform encephalopathy. Histologically, the gold standard method to detect amyloid is the Congo red stain. Congo red dyes the amyloid that appears as a red deposit with normal bright field optics, but also shows "apple green" birefringence with polarised light [16].

Amyloid may be also detected, less specifically, using Thioflavin T stain. Thioflavin $\mathrm{T}$ is a benzothiazole dye that exhibits enhanced fluorescence upon binding to amyloid fibrils and is commonly used to diagnose amyloid fibrils, both ex vivo and in vitro [8].

\section{Immunohistochemistry and Immunofluorescence}

Immunohistochemistry is a method that allows to detect antigens (e.g., proteins) in the cells of a tissue section by exploiting the principle of antibodies binding specifically to antigens in biological tissues. The antigen-antibody reaction can be seen with the light microscope when labels (reporter molecules) connect to the primary, secondary, or tertiary antibodies of a detection system that allows the visualisation of the immune reaction. A variety of labels have been used, most commonly enzymes (e.g., peroxidase, alkaline phosphatase, glucose oxidase). The enzymes in presence of a specific substrate and a chromogen will produce a coloured precipitate at the site of the antigen-antibody reaction [18].

Immunohistochemistry plays a major role in diagnostic neuropathology being able to distinguish the specific antigenic differences between cells and even between infectious agents or pathological cellular inclusions.

The list of antibodies that are used in diagnostic neuropathology is very considerable and continuo- usly increasing. An encompassing review of all the antibodies used in clinical and research activities goes beyond the aims of this review, but it may be useful to mention those that are routinely used for a primary histopathological assessment of the CNS.

For routine diagnostic purposes, currently the most used antibodies for the identification of neurons and their processes are synaptophysin, triple neurofilaments (NF-L, NF-M, NF-H molecular weights) either individually or in various cocktails and Neu-N. Cells of the neuroendocrine system express synaptophysin, chromogranin A and neuron specific enolase. Axons are visualised by antibodies to NF-200 and triple neurofilaments [23].

Classically, Glial fibrillary acidic protein (GFAP) is the most important marker for astrocytes being the main intermediate filament protein in these cells, in addition to vimentin and nestin [13] GFAP is known to be induced upon brain damage or during CNS degeneration, and to be more highly expressed in the aged brain [13]. GFAP may also be an important marker for the diagnosis of tumours of the CNS such as astrocytoma. However, it has some significant limitations. GFAP is not specific for astroglial cells (see below) and, furthermore, it doesn't allow to correlate the degree of its expression and tumour anaplasia or to discriminate neoplastic from reactive cells.

Many antigenic markers are available for oligodendrocytes although no one is specific [3]. Some antigens expressed by oligodendrocytes include myelin basic protein (MBP), myelin associated glycoprotein (MAG) and galactocerebroside (GC) [3]. Other antibodies are used mostly for experimental purposes.

Both resting and activated microglia can be immunohistochemically identified by antibodies to both $\mathrm{CD} 18$ and CD11d. A fraction of microglia and macrophages may react to antibodies such as CD68, lysozyme and MAC (myeloid/histiocyte antigen). However, no antibody is available to discern these functional cell types [23].

Normal and neoplastic ependymal cells are blandly immunoreactive for GFAP and more consistently positive for vimentin.

Normal and neoplastic choroid plexus epithelium is usually immunohistochemically reactive for both low and high molecular weight cytokeratins while the lamina is immunoreactive for collagen IV. Neoplastic choroid plexus epithelium variably expresses GFAP. Meningothelial cells express vimentin and variably cytokeratin. 
Immunofluorescence, as well as immunohistochemistry, is an immunostaining method that combines the use of antibodies and fluorescent molecules for the detection of specific targets in cells and tissues. There are two types of immunofluorescence techniques: primary (or direct) and secondary (or indirect) [16]. Primary immunofluorescence uses a single antibody that is chemically linked to a fluorophore. The fluorophore can be detected via microscopy once the antibody recognises the target molecule and binds to it. Secondary immunofluorescence uses two antibodies; the unlabelled first (primary) antibody specifically binds the target molecule, and the secondary antibody, which carries the fluorophore, recognises the primary antibody and binds to it [16].

This review is the result of our experience working with nervous system. We suggest the application of standard methods that provides an adequate evaluation of the broad category of neuropathological diseases. Additional work and specialised procedures can always be added when necessary [5]. But they should also not be indiscriminately applied to any study just because of uncertainty over what is necessary, particularly when the pressure to do this additional work is being applied by those without the requisite knowledge to logically do so [5]. We are going to show and discuss in details some practical approach in a number of our neuropathological cases.

\section{MATERIALS AND METHODS}

\section{Animals}

Samples were mainly collected from animals euthanised or naturally dead after showing mild to severe neurological clinical signs such as depression, occasional circling and incoordination, seizure and behavioural changes (Table 1). Tissue samples obtained from laboratory animals were submitted to the Laboratory of Anatomic Pathology of the Department of Veterinary Medicine and Animal Production of the University of Naples Federico II for a histological evaluation. The procedures involving laboratory animals were approved by the animal experimentation ethics committee, the European Directive 86/609/ CEE and the European council guidelines included in the European Convention for the Protection of Vertebrate Animals used for Experimental and Other Scientific Purposes.

From 2010 to 2013 we examined brains from 60 bovine, 30 buffaloes, 20 dogs, 10 cats, 5 horses, 40 mice, 2 guinea pigs, 1 rabbit, 1 tiger, 1 chimpanzee.
Animals and tissues included in the study are summarised in Table 1.

\section{Histology}

Samples collected from the CNS, including frontal cortex, basal nuclei, hippocampus, occipital cortex, thalamus, midbrain, cerebellar cortex and spinal cord were fixed in 10\% neutral buffered formalin and embedded in paraffin. Sections were stained first with $\mathrm{H} \& \mathrm{E}$ and subsequently with selected stains such as LFB/cresyl violet (Bio-Optica, Milan), PAS, Congo red (Bio-Optica, Milan) and Bielschowsky (Bio-Optica, Milan). Special stains were performed according to manufacturer's protocols or according to protocol described in histology technique textbook [2].

\section{Immunohistochemistry}

Immunohistochemical stains were performed on selected sections using horseradish peroxidase (HRP) method. Antigen retrieval pretreatments were performed using a HIER (Heat-Induced Epitope Retrieval) citrate buffer pH 6.0 (Bio-Optica, Milan) for $20 \mathrm{~min}$ at $98^{\circ} \mathrm{C}$. Primary antibodies were mouse monoclonal cytokeratin (Clone AE1/AE3, DAKO Italia, Milan), mouse monoclonal vimentin (Clone V9, DAKO Italia, Milan), monoclonal mouse CD3 (Clone F7.2.38), mouse monoclonal CD79 $\alpha$ cy (Clone HM57, DAKO Italia, Milan) and mouse monoclonal $\beta$-amyloid 1-16 (Clone 6E10, Covance Italy, Rome). Working solution were 1:100 for cytokeratin, 1:100 for vimentin, 1:50 for CD3, 1:50 for CD79 $\alpha$ cy and 1:100 for $\beta$-amyloid 1-16. Antigen-antibody binding were detected by a HRP polymer detection kit (MACH1, BioCare, Italy) and the slides were counterstained with haematoxylin.

Histological specimens were examined and photographed with a light microscope (Nikon eclipse E600) associated to a microphotography system (Nikon digital camera DMX1200).

\section{RESULTS}

For diagnostic purposes, in this review, the diseases were classified as inflammatory, metabolic and neoplastic. Tissue changes, either pathologic or physiologic associated with aging, were classified as degenerative disease.

\section{Inflammatory diseases}

Inflammatory diseases were observed in 4 cases out of 26 ( 2 dogs and 2 buffaloes). Inflammatory lesions were mostly associated with viral infections with a non-suppurative encephalitis. The lesions were severe and diffuse with the involvement of meninges (meningoencephalitis) (case no. 11, 15 and 16) and, 
Table 1. Species, breed, sex, age and neurological signs of the animals employed in this study

\begin{tabular}{|c|c|c|c|c|c|}
\hline Case no. & Species & Breed & Sex & Age & Neurological signs \\
\hline 1 & Bovine & Podolic & $\mathrm{F}$ & 19 years & Depression, incoordination \\
\hline 2 & Bovine & Podolic & $\mathrm{F}$ & 12 years & Depression, incoordination \\
\hline 3 & Bovine & Podolic & $\mathrm{F}$ & 11 years & Depression, incoordination \\
\hline 4 & Bovine & Podolic & $\mathrm{F}$ & 9 years & Depression, incoordination \\
\hline 5 & Bovine & Podolic & $\mathrm{F}$ & 7 years & Depression, incoordination \\
\hline 6 & Bovine & Podolic & $\mathrm{F}$ & 9 years & Depression, incoordination \\
\hline 7 & Bovine & Podolic & $\mathrm{F}$ & 11 years & Depression, incoordination \\
\hline 8 & Bovine & Podolic & $\mathrm{F}$ & 12 years & Depression, incoordination \\
\hline 9 & Bovine & Podolic & $\mathrm{F}$ & 10 years & Depression, incoordination \\
\hline 10 & Bovine & Podolic & $\mathrm{F}$ & 10 years & Depression, incoordination \\
\hline 11 & Buffalo & Mediterranean & M & 1 year & Behavioural changes, seizure \\
\hline 12 & Buffalo & Mediterranean & $\mathrm{F}$ & 1 year & Convulsion, paralysis \\
\hline 13 & Dog & German Sheperd & M & 2 years & Paralysis, behavioural changes \\
\hline 14 & Dog & Mixed & M & 10 years & Seizure, paralysis \\
\hline 15 & Dog & Maremman Sheperd & M & 2 years & Behavioural changes, seizure \\
\hline 16 & Dog & Maltese dog & M & 45 days & Behavioural changes, seizure \\
\hline 17 & Dog & Boxer & M & 8 years & Behavioural changes, incoordination, paralysis \\
\hline 18 & Cat & European shorthair & $\mathrm{F}$ & 18 years & Depression, incoordination \\
\hline 19 & Horse & Quarter horse & M & 5 years & Behavioural changes, seizure \\
\hline 20 & Rabbit & & $\mathrm{F}$ & 2 years & Stiff neck \\
\hline 21 & Mouse & & M & 18 months & Behavioural changes \\
\hline 22 & Mouse & & M & 18 months & Behavioural changes \\
\hline 23 & Mouse & & M & 18 months & Behavioural changes \\
\hline 24 & Guinea pig & & $\mathrm{F}$ & 2 years & Depression, incoordination \\
\hline 25 & Tiger & & $\mathrm{F}$ & 16 years & Depression, paralysis \\
\hline 26 & Chimpanzee & & M & 42 years & Depression, paralysis \\
\hline
\end{tabular}

$\mathrm{F}$ - female; $\mathrm{M}$ - male

in 1 case (case no. 12) even of the spinal cord (meningoencephalomyelitis). Perivascular cuffing with the collection of inflammatory cells around blood vessels was a constant pathological hallmark. Viral infections have a marked tropism for neurons, thus a widespread neuronal loss and degeneration were common features with necrosis, glial nodules with neuronophagia and central chromatolysis (Fig. 3). In a case of canine distemper virus infection (case no. 15), demyelinated plaques and viral nuclear inclusion body were also found (Fig. 4).

\section{Metabolic diseases}

Hepatic encephalopathy was diagnosed only in 1 case (case no. 14) out of 26. A 10-year-old mixed dog showing neurological signs and paralysis was referred to the teaching hospital of the Department of Veterinary Medicine and Animal Production of the University of Naples Federico II. The owner decided for the euthanasia after the worsening of the dog's conditions. Post mortem evaluation of CNS showed no meaningful lesions. Histologically, there were white matter vacuolisation with numerous vacuoles of varying size (spongy state) due to a cytotoxic oedema. Additionally, there were scattered astrocytes with swollen nuclei, marginalised chromatin and a clear centre referred to as Alzheimer type II cells that are indicative of a metabolic encephalopathy (Fig. 5).

\section{Neoplastic diseases}

Nervous system tumours were diagnosed in 2 cases out of 26 , both in dogs. The first case (case no. 17) was an 8-year-old dog, a boxer, that went for a medical examination for neurological signs such 


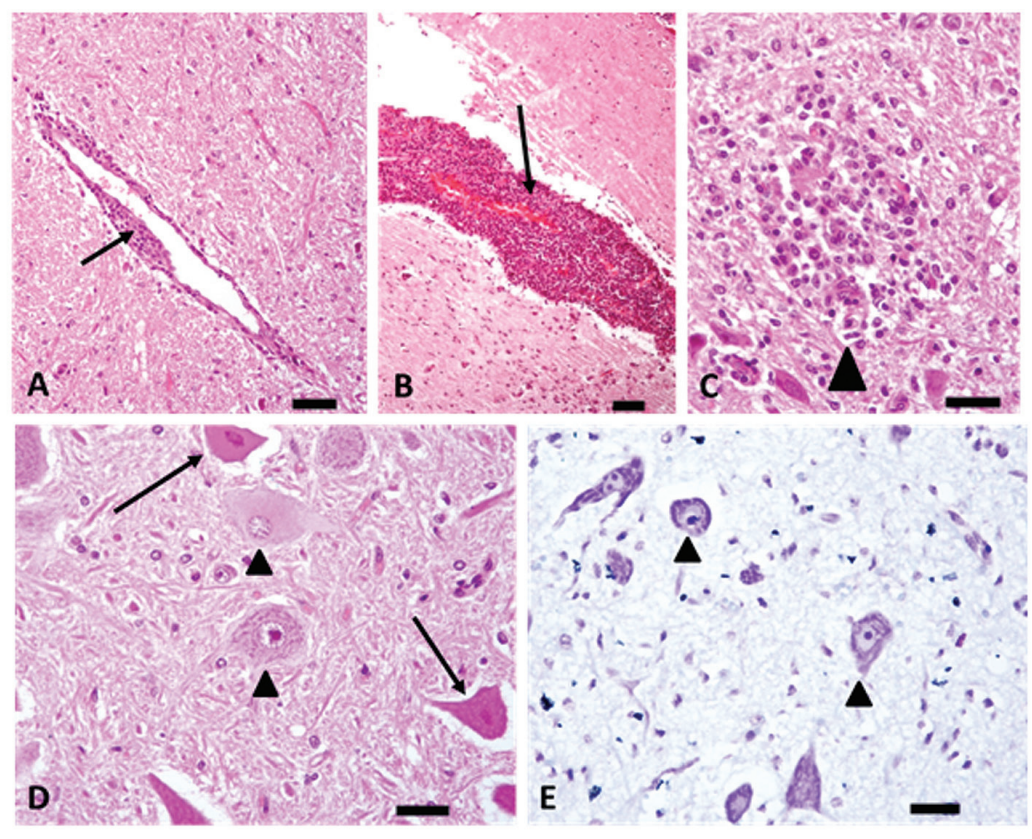

Figure 3. Meningoencephalomyelitis. Mediterranean Italian buffalo; A. Brainstem. Mild limphoplasmocytic vasculitis (arrow); haematoxylin-eosin (H\&E); Bar $=100 \mu \mathrm{m}$; B. Cerebellum. Leptomeningitis. Perivascular inflammation of meninges (arrow); H\&E; Bar $=80 \mu \mathrm{m}$; C. Cerebellar cortex. Focal microglial cell nodule with neuronophagia (arrow head); H\&E; Bar $=100 \mu \mathrm{m} ;$ D. Spinal cord, ventral horn. Necrotic and hypereosinophilic neurons (arrows). Degenerating neurons show swollen cytoplasm and peripheral or total chromatolysis (arrow heads); H\&E; Bar $=20 \mu \mathrm{m}$; E. Spinal cord, ventral horn. Central chromatolysis with loss of Nissl body in the centre of the cell; cresyl echt violet; Bar $=20 \mu \mathrm{m}$
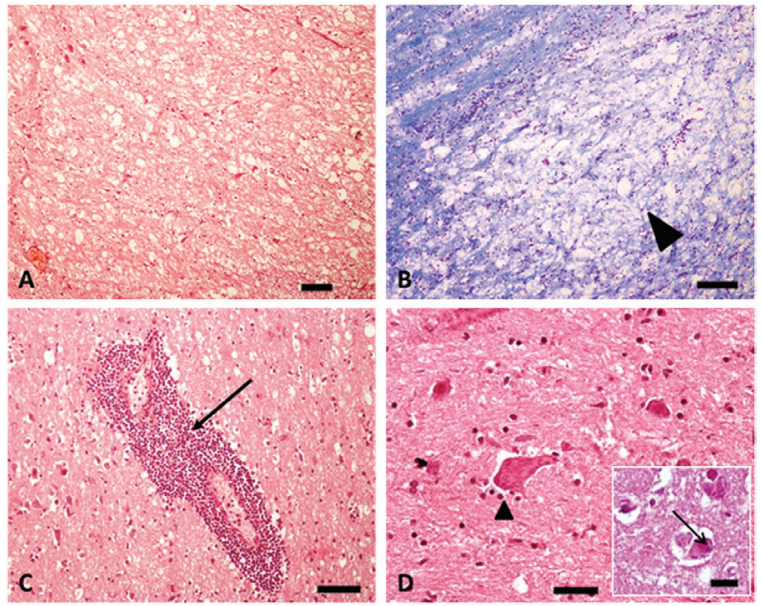

Figure 4. Canine distemper virus, dog; A. Medulla. Acute demyelination with spongy state of white matter; haematoxylin-eosin (H\&E); Bar $=100 \mu \mathrm{m}$; B. Medulla. Demyelinated plaque with intact myelin stained blue (arrow head). Luxol fast blue-cresyl violet; Bar $=100 \mu \mathrm{m}$; C. Medulla. Severe polioencephalitis with perivascular cuffs (arrow); H\&E; Bar $=100 \mu \mathrm{m}$; D. Medulla. Necrotic neurons with shrunken and ipereosinophilic cytoplasm. A mild gliosis is also present (arrow head); H\&E; Bar $=20 \mu \mathrm{m}$. Inset: Intranuclear eosinophilic viral inclusion body (arrow); H\&E; Bar $=10 \mu \mathrm{m}$.

as depression and behavioural change. Computed tomography analysis detected an intraventricular space-occupying mass suggestive of a meningioma. Neuropathological post mortem assessment confirmed the presence of a tumour. Grossly, the mass was well demarcated, firm and whitish. On microscopic examination, there were whorls of spindle cells with

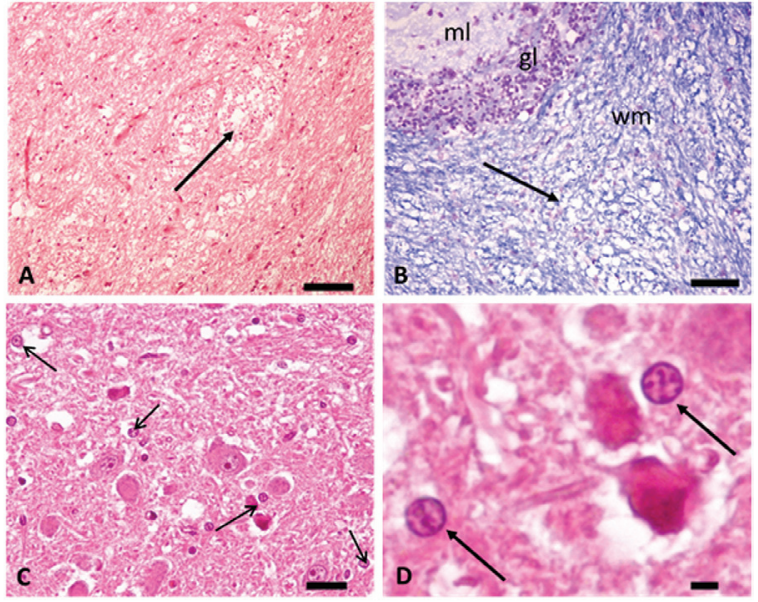

Figure 5. Hepatic encephalopathy, $\operatorname{dog}(\mathbf{A}, \mathbf{B})$. Cerebellum. Spongy degeneration Massive white matter vacuolisation (arrows). Haematoxylin-eosin (H\&E) (A) and luxol fast blue (B); Bar $=100 \mu \mathrm{m}$; $\mathrm{ml}$ - molecular layer; gl — granular cell layer; wm — white matter; C. Basal nuclei area. Many single Alzheimer type II cells with swollen nuclei and marginalized chromatin (arrows); H\&E; Bar $=50 \mu \mathrm{m}$; D. Particular of Alzheimer type II cells with clear centre and marginalised chromatin (arrows); $\mathrm{H} \& \mathrm{E} ; \mathrm{Bar}=10 \mu \mathrm{m}$.

scant eosinophilic cytoplasm and mild nuclear atypia. Immunohistochemical analysis demonstrated the immunoreactivity of neoplastic cells to vimentin, but they were negative for cytokeratin (Fig. 6). The tumour was histologically classified as a low grade transitional meningioma. The second case (case no. 13) was a 2 year-old-dog, German shepherd, with a meta- 

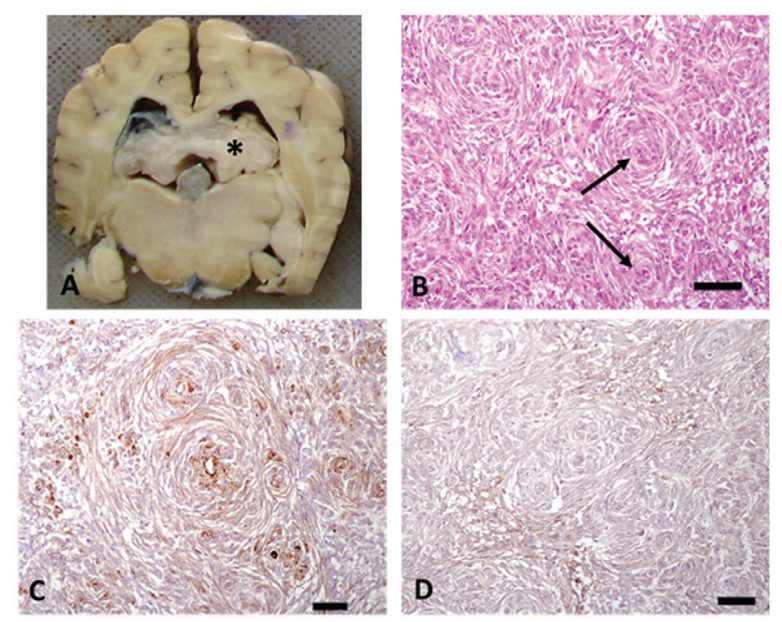

Figure 6. Meningioma, dog; A. Intraventricular, expansive, solid grey mass (asterisk); B. Histology of the mass in panel A. Syncytial cell clusters and concentric whorls of neoplastic cells (arrows); Haematoxylin-eosin; Bar $=50 \mu \mathrm{m} ;$ C. Immunoreactivity of neoplastic cells to vimentin; Bar $=20 \mu \mathrm{m}$; D. Neoplastic cells are negative for cytokeratin; Bar $=50 \mu \mathrm{m}$.

static multicentric lymphoma. The dog died naturally for cardiac complications. A necropsy examination allowed to detect a single, submeningeal space-occupying mass localised on the occipital lobe that was pinkish to red for the presence of haemorrhages, soft and poorly defined. Histological, neoplastic cells were either lymphoblastic or more mature lymphocytes characterised by large, round to oval and basophilic nuclei with prominent nucleolus and scant cytoplasm. Areas of haemorrhages and necrosis were also found. Immunoreactivity to CD79 $\alpha$ suggested a B cell canine lymphoma (Fig. 7).

\section{Degenerative diseases and ageing}

All the aged animals included in this study (13 out of 26) showed aged-related lesions. The species included in the study were bovine (cases no. 1-10), a cat (case no. 18), a tiger (case no. 25) and a chimpanzee (case no. 26). The severity of the lesions varied depending on the species and, most of all, on the age. A constant and conspicuous feature in the brain of old animals was the gliosis and the increased number of glial cells around neurons (satellitosis) (Fig. 8). Another typical and constant finding was the accumulation of PAS positive and granular material within the neurons consisting of lipofuscin granules. The amount of lipofuscin was large with a wide distribution in cerebral cortex, hippocampus, basal nuclei, occipital cortex and cerebellar cortex (Fig. 9).
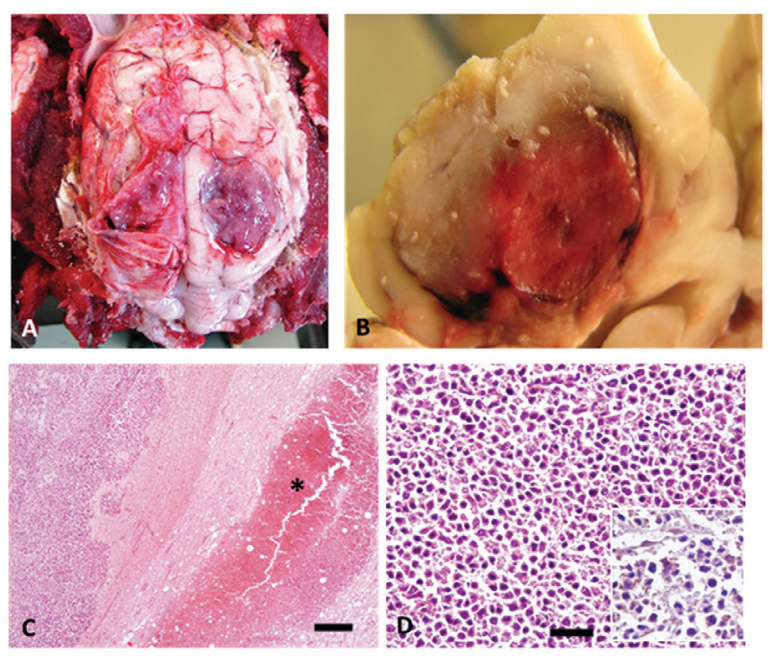

Figure 7. Metastatic B cell lymphoma, dog; A. Pinkish, soft, poorly defined single mass in occipital lobe; B. Transverse section of the mass; C. Histology from the mass in panels A and B. Diffuse infiltration of the neuropil of neoplastic cells. At the periphery of the lesion there is an area of haemorrhage (asterisk); Haematoxylineosin (H\&E); Bar $=200 \mu \mathrm{m}$; D. Neoplastic cells have a large, round to angular and densely basophilic nuclei; H\&E; Bar $=50 \mu \mathrm{m}$; Inset: tumour cells are strongly positive to CD79 $\alpha$ cy.

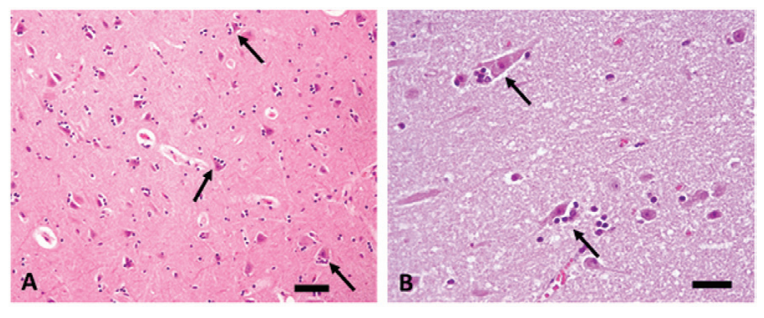

Figure 8. Satellitosis. A. Forebrain, tiger. Cluster of oligodendrocytes surround neurons (arrows); Haematoxylin-eosin (H\&E); Bar $=50 \mu \mathrm{m}$; B. Forebrain, cow. Rings and clusters of oligodendrocytes near degenerating neuron cell body (arrows); H\&E; $\mathrm{Bar}=10 \mu \mathrm{m}$.

Cerebrovascular amyloidosis was also detected (case no.1 and no. 18), affecting both leptomeningeal and parenchymal arterioles and capillaries. Amyloid protein was deposited in the tunica media of the vessel walls mostly in a continuous ring-shaped aspect (Fig. 10).

Modified Bielschowsky stain allowed to detect neurofibrillary tangles that appeared as flame-shaped accumulation of argyrophilic fibrillary material that fills the cell body or it is distributed around the perikarya of neurons (Fig. 11A-C). Neurofibrillary tangles were a very rare finding, typically affecting the hippocampus of the oldest animals (case no. 1, no. 18 and no. 26). 


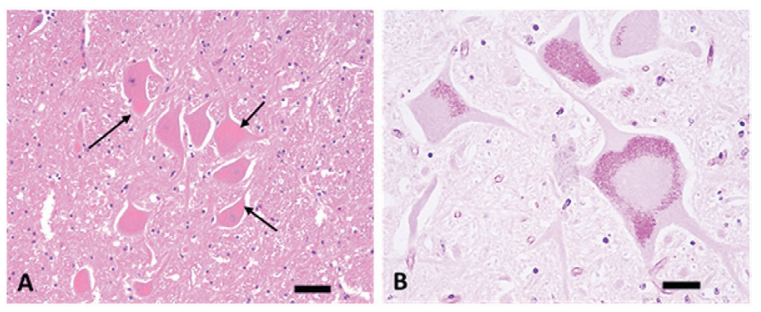

Figure 9. Lipofuscin storage, pons, chimpanzee; A, B. A large amount of light brown granular deposit in neuronal body (arrows). Haematoxylin-eosin; Bar $=50 \mu \mathrm{m}$. The same granular material is strongly periodic acid Schiff positive; Bar $=10 \mu \mathrm{m}$.

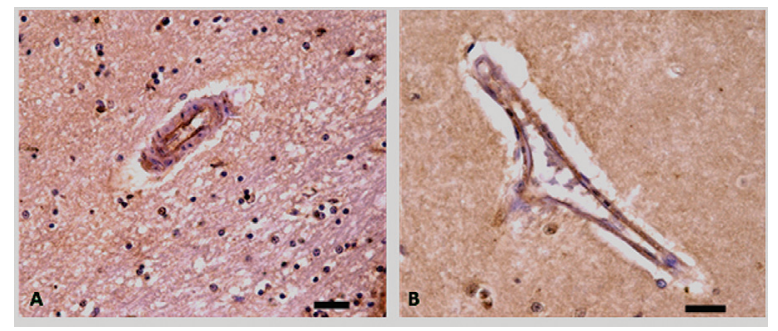

Figure 10. Cerebrovascular amyloidosis, brain, old cattle; A, B. $\beta$-amyloid deposition in the wall of a vessel. Immunohistochemistry, horseradish peroxidase method for $\beta$-amyloid protein, Mayer's haematoxylin counterstained; Bar $=10 \mu \mathrm{m}$.

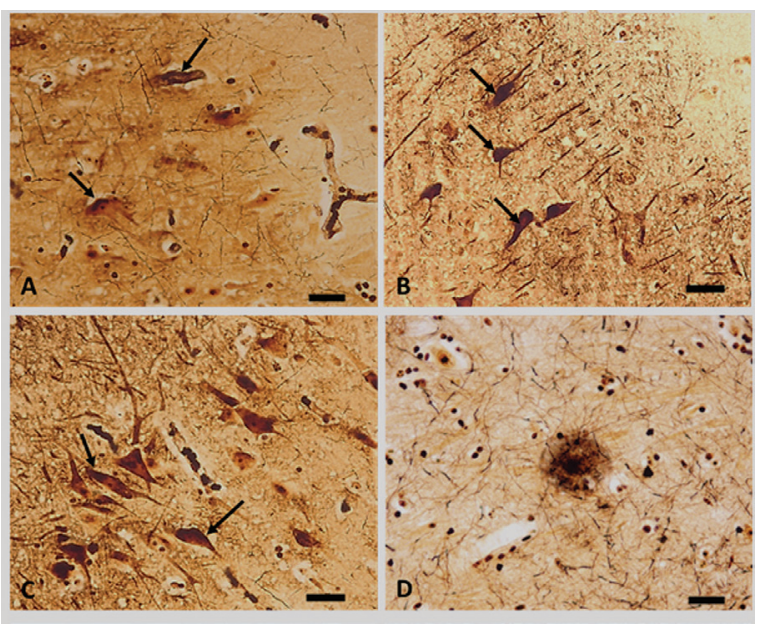

Figure 11. A, B, C. Ageing related lesions. Neurofibrillary tangle, hippocampus, chimpanzee. Flame-shaped filaments aggregates within the perikarya of a neuron (arrows). Bielschowsky stain (Bar $=50 \mu \mathrm{m}$ for $\mathbf{A}$; Bar $=10 \mu \mathrm{m}$ for C); D. Senile plaque, hippocampus, cow. A senile plaque with amyloid core. Bielschowsky stain; Bar $=10 \mu \mathrm{m}$.

Modified Bielschowsky stain also highlighted scattered, diffuse senile plaques that appear as a dark brown to black focal aggregation of degenerating neurites and glial cells around a central extracellular

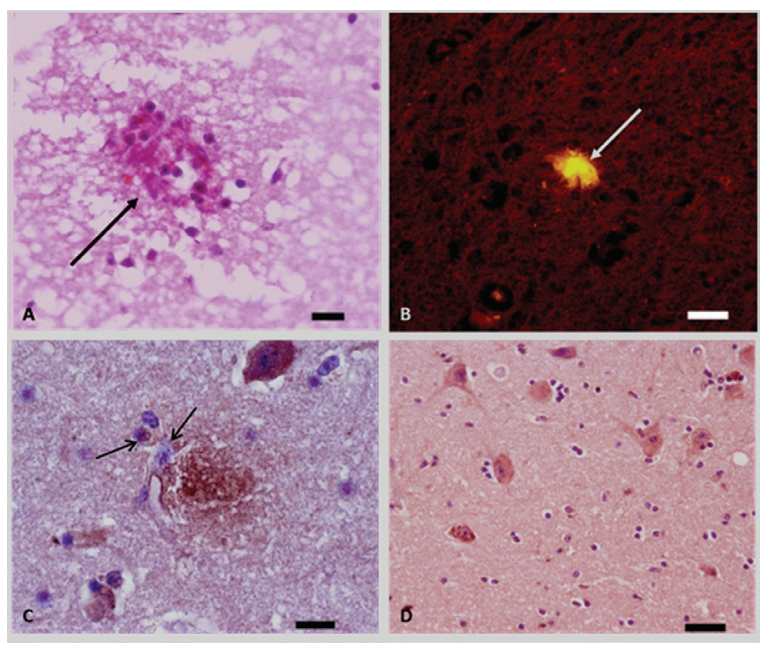

Figure 12. Ageing related lesions, hippocampus; $\operatorname{cow}(\mathbf{A}, \mathbf{B})$;

Senile plaque with an amyloid core stained with Congo red (arrow). Congo red without polarisation $(\mathbf{A})$ and seen with a fluorescent microscope (B) $(B a r=10 \mu \mathrm{m})$; C. Senile plaque of a diffuse type showing immunopositivity for $A \beta$ 1-16 antibody and entrapping normal neurons (arrow); D. Immunoreactivity for $A \beta$ 1-16 antibody is strong and diffuse in neuronal bodies. Immunohistochemistry, horseradish peroxidase method for $\beta$-amyloid protein, Mayer's haematoxylin counterstained (Bar $=5 \mu \mathrm{m}$ for $\mathrm{C}$ and Bar $=10 \mu \mathrm{m}$ for D).

amyloid core (Fig. 11D). The $\beta$-amyloid central core of senile plaques was also observed with Congo red stain using both a light and a fluorescent microscope (Fig. 12A, B) and with $\beta$-amyloid 1-16 immunohistochemical staining (Fig. 12C). Senile plaques affected oldest animals and were located mostly in the hippocampus.

A strong immunoreactivity to $\beta$-amyloid was also observed in neurons without associated plaques (case no. 10) (Fig. 12D).

In 1 case (case no. 26) concentric calcium deposits in the tunica media of the smaller arteries were also found (Monckeberg's arteriosclerosis or medial calcific sclerosis). The foci of dystrophic calcification involved partially or diffusely the circumference of the tunica media, but were not associated to luminal narrowing or closure of the lumen of the vessels nor to atherosclerotic plaques (Fig. 13A, B).

\section{Artefacts}

The artefact can occur very often during the examination of CNS and the pathologists have to be very careful in the interpretation of the result to avoid misinterpretation.

In our cases, very common artefacts were single or clusters of strongly basophilic and shrunken neurons 


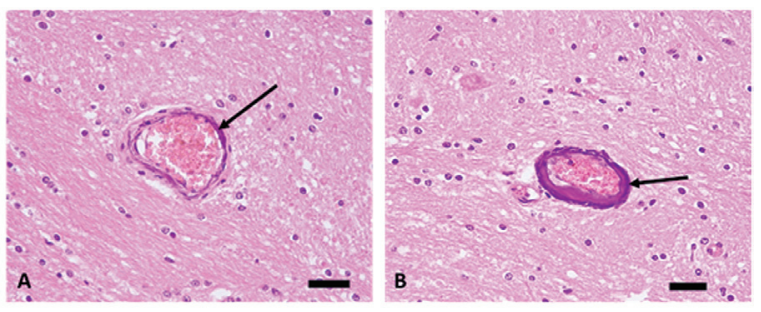

Figure 13. Medial calcific sclerosis, basal ganglia, chimpanzee (A, B) and calcium deposits within the tunica media of arteries (arrows); haematoxylin-eosin; Bar $=50 \mu \mathrm{m}$.

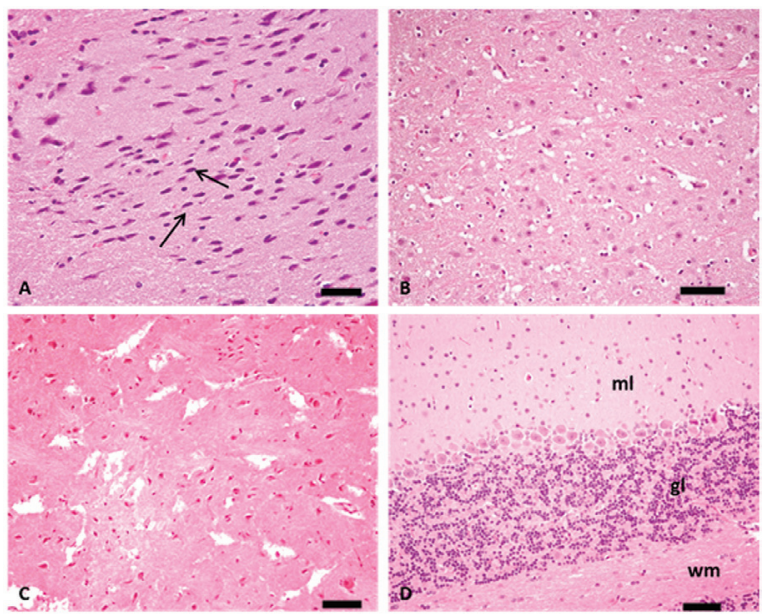

Figure 14. Artefacts. A. Mouse, cerebral cortex. Dark neurons (arrows). Haematoxylin-eosin (H\&E); Bar $=50 \mu \mathrm{m}$; B. Cat, cerebral cortex. Post-mortem autolysis with vacuolation of grey matter; H\&E; Bar $=50 \mu \mathrm{m} ;$ C. Dog, cerebral cortex. Vacuolation and clefts in the grey matter indicating that the cadaver was frosted before the necropsy; H\&E; Bar $=50 \mu \mathrm{m}$; D. Mouse, cerebellar cortex. Mild post-mortem lysis of cerebellar granule layer; $\mathrm{ml}$ - molecular layer; $\mathrm{gl}$ — granular layer; wm — white matter; $\mathrm{H} \& \mathrm{E} ; \mathrm{Bar}=50 \mu \mathrm{m}$.

known as "dark neurons" (Fig. 14A). Very common finding were also the vacuolation both of the grey and white matter and the presence of "clefts" in the cerebral parenchyma, mostly observed in specimen collected from frosted cadavers. The vacuoles were irregular in shape and size and they were not well defined (Fig. 14B, C). Depletion of granule cell of the cerebellar cortex was also seen with the reduction of the number of these cells often associated to perineuronalvacuolation of Purkinje cells (Fig. 14D).

\section{DISCUSSION}

A proper approach to neuropathology is essential for diagnostic purposes. The processes of collecting and sampling tissues as well as the macroscopic and microscopic evaluation of CNS have to be carefully performed in order to obtain useful information for a better understanding of the lesions, of the pathological features and, to an extent, of the disease. Removal and successive handling of the brain and the spinal cord are decisive points that could affect the final outcome. Central nervous tissues have to be handle with extreme care in order to avoid any artefactual changes. The preparation of tissue for histological study is a multi-step process in which potential loss of quality and the introduction of artefacts can occur during each step. Knowledge of the process and the potential pitfalls at each step will incomparably serve the investigator [11].

Tissues have to be immersed in an adequate volume of fixative solution as soon as possible, since post-mortem-degeneration progresses rapidly in nervous tissue and causes the loss of structural details in histological section compromising the diagnosis. Alternatively to immersion, perfusion fixation has been used as a technique in neuroscience research laboratories for years, and it is often recommended for generating optimal tissue sections for microscopic examination [5]. Perfusion fixation is not always technically easy to perform, particularly when done in large animal species or in large numbers of rodents [5]. Perfusion fixation has the advantage to often reduce the level of artefactual change, but it does not completely eliminate this problem and failure [5]. A procedure not correctly performed can potentially result in tissue sections of poorer quality than those that are routinely fixed by immersion [5]. Since these aforementioned variables will have a major influence on the level of artefact in histological sections, the utility of perfusion fixation should be addressed on a case-by-case basis by the laboratory in which the procedure will be carried out [5].

As some authors pointed out, a thorough evaluation of the CNS depends mostly on a sufficient number of sections from brain and spinal cord [1], besides a representative sampling that involve the principal areas of the brain and spinal cord and all the areas affected by lesions macroscopically evident or suggested by a neurological and clinical examination.

The histological evaluation of CNS tissues is generally performed with sections stained with $\mathrm{H} \& \mathrm{E}$ that shows the basic morphology of tissues and allows the detection of lesions in almost all cases. However, special stains are a fundamental tool in comparative neuroanatomy and neuropathology to better characterize the structure and lesions detected with H\&E. 
Special histochemical stains such as PAS, GMS, Gram or Ziehl-Nelseen are essential to highlight the fungi or the bacteria that are responsible of CNS infections. Routine histopathologic methods employed in general pathology studies are needed to identify the complete range of possible neuropathologic changes; once identified, many of these changes can be better defined by specialised techniques, such as immunohistochemistry, to confirm cell types involved [5].

Immunohistochemical staining can also be useful for a specific etiological diagnosis, especially in the case of viral infection.

Neurodegenerative disorders include a wide range of diseases characterised by selective degeneration, loss of cells and cell components (e.g. axons or myelin sheaths), intracellular storage and/or extracellular deposits. Lesions are often not visible on a macroscopic examination and the specific patterns can only be demonstrated by histochemical special stains such as LFB/cresyl violet, silver impregnation (modified Bielschowsky, Bodian, GAL method) or Fluoro-Jade C, PAS and Congo red are necessary to detect storage material within neurons (e.g. lipofuscin or Lafora bodies) or in the cerebral parenchyma (e.g. amyloid). A fluorescent microscope is useful for identification of autofluorescent degenerating neurons either in H\&E-stained slides or in Fluoro Jade C-stained sections when increased sensitivity is needed [6].

Primary and secondary tumours of the CNS can be recognised and diagnosed macroscopically and histologically. H\&E stain is generally sufficient for the recognition of the general features of the neoplasia and the malignancy aspects. However, immunohistochemical staining is often necessary to confirm the histological diagnosis and grading of the neoplasia either with cell-specific antigenic markers or proliferation markers.

In our cases the most common neuropathological changes were those related to aging and inflammation rather than to metabolic disorders or neoplasia. Tissue changes associated with old age were consistent although it is still debatable whether call them pathologic lesions or simply physiologic age-related alteration. Interestingly, we did not observed evidences of vascular disorders such as stroke or ischaemia which are quite rare in animals compared to humans [23]. Inflammatory diseases were mostly associated to viral infections and no evidences of autoimmune diseases were found. Primary nervous system tumours were quite rare so it was not possible to report their incidence and the predisposition for species, age, sex, breed or histological type.

\section{CONCLUSIONS}

Finally, this review emphasised several practical aspects for a neuropathological assessment of the CNS. This simple guide may be used for a correct approach to processes and techniques that have to be carefully mastered in order to provide high quality material for an adequate evaluation of the lesions and to win the challenges that a pathologist has to engage working with CNS.

\section{REFERENCES}

1. Bolon B, Anthony Douglas C, Butt M, Dorman D, Green MV Little PB, Valentine WM, Weinstock D, Yan J, Sills RC (2008) "Current Pathology Techniques" Symposium Review: Advances and Issues in Neuropathology. Toxicol Path, 36: 871-889.

2. Culling CFA (1975) Handbook of histopathological and histochemical techniques. 3rd Revised Edition. Butterworth-Heinemann, Oxford, UK.

3. Deng W, Poretz RD (2003) Oligodendroglia in developmental neurotoxicity. NeuroToxicology, 24: 161-178

4. Fix A, Sand Garman RH (2000) Practical aspects of neuropathology: a technical guide for working with the nervous system. Toxicol Pathol, 28: 122-131.

5. Houle CD (2011) Neuropathology standards: What constitutes an optimal histomorphologic evaluation of the nervous system in general toxicity studies. Toxicol Pathol, 6: 1010-1012.

6. Jordan WH, Young JK, Hyten MJ, Hall DG (2011) Preparation and analysis of the central nervous system. Toxicol Pathol, 39: 58-65.

7. Kepes JJ, Oswald O (1991) Tissue artifacts caused by sponge in embedding cassettes. Am J Surg Pathol, 15: 810-812.

8. Khurana Ritu R, Coleman CC, Ionescu-Zanetti C, Carter SA, Krishna V Grover RK, Roy R, Singh S (2005) Mechanism of thioflavin T binding to amyloid fibrils. J Struct Biol, 151: 229-238.

9. Manlow A, Munoz DG (1992) A non-toxic method for the demonstration of gliosis. J Neuropathol Exp Neurol, 51: 298-302.

10. Mannoji H (1986) A specific histochemical marker (lectin Ricinuscommunis agglutinin-1) for normal human microglia, and application to routine histopathology. Acta Neuropathol, 71: 341-343.

11. McAuliffe WG (2013) Routine histology techniques for the developing and adult central nervous system. Methods Mol Biol, 1018: 289-300.

12. Meikle ADS, Martin AH (1981) A rapid method for removal of the spinal cord. Stain Technol, 6: 235-237.

13. Middeldorp J, Hol EM (2011) GFAP in health and disease. Prog Neurobiol, 93: 421-443.

14. Mitchell J, Best N, Sundstrom LE, Wheal HV (1993) The use of sodium sulphide-fixed brain tissue for immunocytochemical staining of activated microglia and reactive astrocytes Histochemistry, 99: 91-94.

15. Mugnaini E, Dahl A (1983) Zinc-aldehyde fixation for light-microscopic immunocytochemistry of nervous tissues. J Histochem Cytochem, 31: 1435-1438.

16. Paciello O, Papparella S (2009) Histochemical and immunohistological approach to comparative neuromuscular diseases. Folia Histochem Cytobiol, 47: 143-152.

17. Pintozzi RL (1978) Modified Grocott' smethenamine silver nitrate method for quick staining of Pneumocystis carinii. J Clin Pathol, 31: 803-805.

18. Ramos-Vara JA (2005) Technical aspects of immunohistochemistry. Vet Pathol, 42: 405-426.

19. Ranjan A, NathMallick B (2012) Differential staining of glia and neurons by modified Golgi-Cox method. J Neurosci Meth 209: 269-279.

20. Sillevis-Smitt $P$, van der Loos C, Vianney de Jong JMB, Troost D (1993) Tissue fixation methods alter the immunohistochemical demonstrability of neurofilament proteins, synpatophysin, and glial fibrillary acidic protein in human cerebellum. Acta Histochem, 95: 13-21.

21. Summers BA, Cummings JF, de Lahunta A (1995) Veterinary neuropathology. Mosby, St. Louis, USA.

22. Uchihara T (2007) Silver diagnosis in neuropathology: principles, practice and revised interpretation. Acta Neuropathol, 2007; 113: 483-499.

23. Vandevelde M, Higgins RJ, Oevermann A (2012) Veterinary neuropathology, essentials of theory and practice. John Wiley \& Sons, Chichester, UK.

24. Williams RS (1983) Golgi's method of staining nerve cells. J Neuropathol Exp Neurol, 42: 210-212. 